\title{
Platelet impedance aggregation in whole blood and its inhibition by antiplatelet drugs
}

\author{
IJ MACKIE, R JONES, ${ }^{*}$ SJ MACHIN
}

\begin{abstract}
From the Department of Haematology, Middlesex Hospital Medical School, London W1, and the *Department of Medicine, University College Hospital, London W1
\end{abstract}

SUMMARY Platelet aggregation was studied in citrated whole blood by an electrical impedance method. Blood samples from normal volunteers were studied with the aim of finding a suitable method for the routine study of samples from patients. An erratic tracing and low maximum aggregation were seen in samples with a high normal haematocrit. Optimal aggregation was seen when blood was diluted to a haematocrit of $\cdot 300$; isotonic saline was a better diluent than platelet poor plasma. No appreciable differences were seen when the platelet count was diluted down to $50 \times 10^{9} / 1$, after which there was a progressive reduction in response. Dose response curves were obtained, and normal ranges for ADP, collagen, and sodium arachidonate were determined. Acetylsalicylic acid had a more pronounced effect on ADP aggregation than on collagen. Prostacyclin (Epoprostenol) and the synthetic prostacyclin analogue ZK 36,374 both showed dose dependent inhibition of aggregation, but the duration of effect of the latter was much longer $(>6$ h).

For the last 20 years platelet aggregation has been studied extensively by optical methods using platelet rich plasma and turbidometric devices. ${ }^{\prime}$ Although of proved diagnostic value, these systems have certain limitations. Centrifugation is required for sample preparation so that red cells, white cells, and some of the heavier platelets are removed from the final platelet suspension to be tested. These other blood cells are probably important modulators of platelet function in vivo since they actively take up adenine nucleotides, synthesise and release regulatory prostaglandins, and may preferentially bind prostacyclin..$^{2-4}$ Substances that regulate platelet function such as prostacyclin and cyclic AMP may degrade to biologically inactive products in the time required for platelet rich plasma preparation.

The recent innovation of electronic aggregation by the impedance method in whole blood circumvents most of these problems. ${ }^{5}$ With this system, blood may be analysed immediately after sampling without centrifugation and aggregation can be investigated in the presence of all blood elements. Most reports describe whole blood platelet aggregation in blood from laboratory animals; we have studied the application of this method to human blood in a routine coagulation laboratory.

Accepted for publication 24 March 1984

\section{Material and methods}

Blood was obtained from apparently healthy adult human volunteers, who had not taken any anti platelet drugs for at least two weeks before sampling. Blood was anticoagulated with $3 \cdot 13 \%$ tri-sodium citrate, nine parts blood to one part anticoagulant. A full blood count and platelet count were performed using a Coulter S Plus IV (Coulter Electronics Ltd, Luton, Beds). Normal subjects were also studied after ingesting varying doses of acetylsalicylic acid.

\section{WHOLE BLOOD PLATELET AGGREGATION}

Whole blood platelet aggregation was performed in citrated blood using the Chronolog Model 540 whole blood aggregometer (Coulter Electronics Ltd, Luton, Beds). A $975 \mu \mathrm{l}$ sample of blood (neat or diluted with platelet poor plasma or isotonic saline) was warmed for $2-3 \mathrm{~min}$ at $37^{\circ} \mathrm{C}$ in the machine heater block and then stirred with a Teflon coated stir bar in a non-siliconised glass cuvette $10 \times 44$ $\mathrm{mm}$ after inserting the impedance electrode. The calibration and gain controls were used to calibrate a Rikadenki R302 chart recorder in ohms. Twenty five microlitres of aggregating reagent was added and the change in impedance (ohms) recorded at a paper speed of $2 \mathrm{~cm} / \mathrm{min}$, giving an aggregation 
trace. The following aggregating reagents were studied (final concentrations in whole blood): 1-25 $\mu \mathrm{M}$ ADP (Sigma Chemical Co) 2.5-125 $\mu \mathrm{M}$ adrenaline (Immuno Ltd), 1-25 $\mu \mathrm{g} / \mathrm{ml}$ collagen (Hormon Chemie, Munchen), 0.5-1 mM sodium arachidonate (Sigma Chemical Co). Dose response curves were produced for each reagent. The possibility of spontaneous aggregation was also studied by stirring undiluted blood at $37^{\circ} \mathrm{C}$ for $15 \mathrm{~min}$ and recording any change in impedance. Aggregation was performed at stirrer speeds varying between 100 and $1200 \mathrm{rpm}$ on the same sample of blood, using a fixed final concentration of one agonist. After completion of impedance aggregation the electrodes were scrupulously cleaned to remove all aggregated cells using isotonic saline and by gentle cleaning with a tissue.

\section{QUANTITATION}

Rate of aggregation was assessed by measuring the angle between the base line and the linear portion of the aggregation curve; thus increased aggregation rate was shown by an increase in angle. Extent of aggregation was measured as the maximum height of response in chart paper units. This could also be expressed in ohms, since 10 units equalled $5 \mathrm{ohms}$.

\section{INFLUENCE OF HAEMATOCRIT}

Aggregation was also performed on undiluted whole blood and after diluting with isotonic saline or platelet poor plasma to reduce the haematocrit. Both normal and polycythaemic samples were studied to create a wide range of haematocrits between $\cdot 100$ and $\cdot 600$.

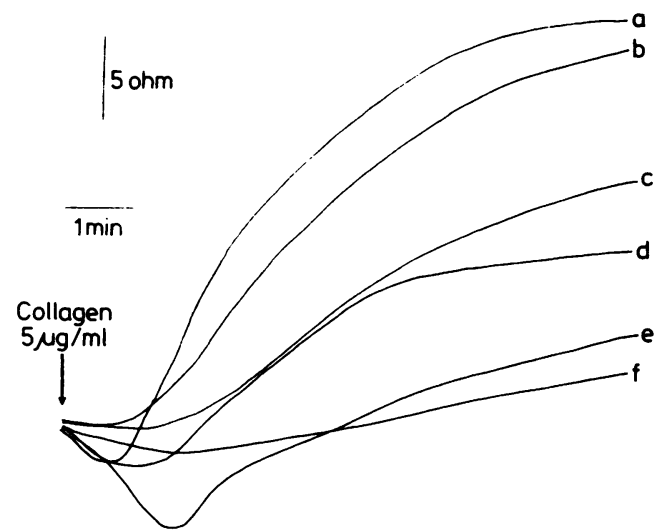

Fig. 1 Influence of haematocrit and diluent. Whole blood platelet aggregation to $5 \mu \mathrm{g} / \mathrm{ml}$ collagen at various haematocrits prepared by dilution with saline (a) $\cdot 300,(b) \cdot 100,(d) \cdot 350,(e) \cdot 420$ (undiluted), ( $f$ ) .050 , or with platelet poor plasma (c) $\cdot 100$.

\section{PLATELET COUNT}

Two thrombopenic subjects (platelets $<25 \times 10^{9} / \mathrm{l}$ ) were bled to study the effect of platelet count. Whole blood platelet aggregation in these subjects was barely detectable using collagen or ADP. Varying amounts of platelet rich plasma from normal (blood group compatible) donors were diluted in citrated whole blood from thrombopenic patients and the volume corrected with platelet poor plasma to give a range of final platelet counts, at a constant haematocrit of $\cdot 300$. Alternatively, a $975 \mu \mathrm{l}$ aliquot of thrombopenic blood was allowed to settle for 20 min and a volume of platelet rich plasma was removed and replaced with platelet rich plasma from a normal donor.

\section{REPRODUCIBILITY}

Precision was studied using normal blood and repeating aggregation to one reagent on the same sample.

\section{INHIBITORS}

The effects of adding prostacyclin (Epoprostenol, Wellcome Pharmaceuticals) and the stable prostacyclin analogue ZK 36,374 (Schering Chemicals Ltd) were studied by adding varying concentrations to whole blood before the addition of aggregating reagents. The dose $\left(\mathrm{ID}_{50}\right)$ causing a $50 \%$ reduction in response to agonists was estimated.

\section{Results}

As expected, low stirring speeds produced poor aggregation responses; optimal aggregation occurred at about $600 \mathrm{rpm}$. Higher stirring speeds showed a gradual reduction in the total impedance change.

Changes in haematocrit produced dramatic effects on impedance (Fig. 1). High haematocrits caused erratic tracings and often minimal aggregation with poor precision, while large dilutions with saline also caused a reduction in platelet count, which also decreased the aggregation response. Dilution to a haematocrit of $\cdot 300$ appeared to be optimal; this gave a better wave of aggregation and a less erratic trace than seen with neat whole blood while causing a minimal decrease in platelet numbers. This also enabled standardisation of whole blood aggregation from different subjects. Saline appeared to be the diluent of choice, giving a steeper and more regular trace than platelet poor plasma.

Reduction of the platelet count by dilution of platelet rich plasma in thrombopenic blood showed no significant changes in aggregation down to platelet counts of about $50 \times 10^{9} / 1$. Below this value a progressive reduction in the response was seen (Fig. 2). 


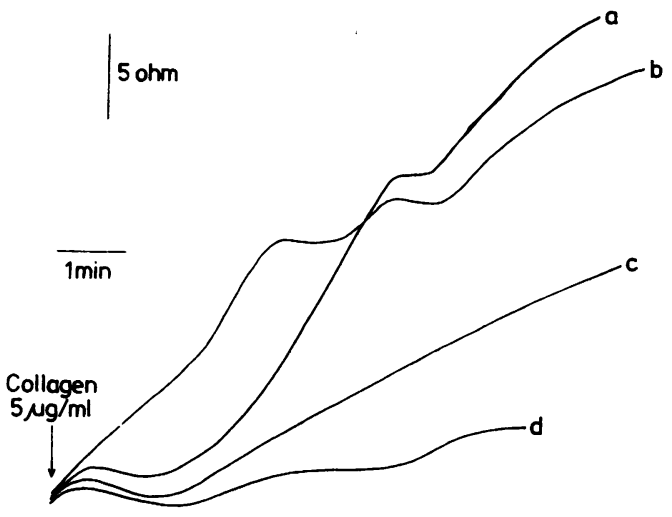

Fig. 2 Effect of platelet count. Aggregation to 5 $\mu \mathrm{g} / \mathrm{ml}$ collagen at a constant haematocrit of $\cdot 300$ prepared by dilution with platelet poor plasma and platelet rich plasma to give various platelet counts: (a) 50 , (b) 100 , (c) 25, (d) $13 \times 10^{4} / l$.

Reproducibility testing showed a coefficient of variation between $15 \%$ and $20 \%$, depending on the sample used and the dose of aggregating reagent; poor aggregation waves tended to be less reproducible.

Good aggregation responses could be obtained with collagen at concentrations of $1-5 \mu \mathrm{g} / \mathrm{ml}$ (Fig. 3). Aggregation with ADP required doses about 10 times higher than those routinely used for platelet rich plasma aggregation (Fig. 3). Only a single wave of aggregation was observed at ADP concentrations causing any impedance change; concentrations of 12.5 and $25 \mu \mathrm{M}$ appeared to be most useful for study. Aggregation with adrenaline was extremely poor, giving low impedance changes even at 125 $\mu \mathrm{M}$. Normal ranges for ADP, collagen, and sodium arachidonate were established and are shown in the Table. Normal donor blood showed no appreciable differences (other than normal variation) when the

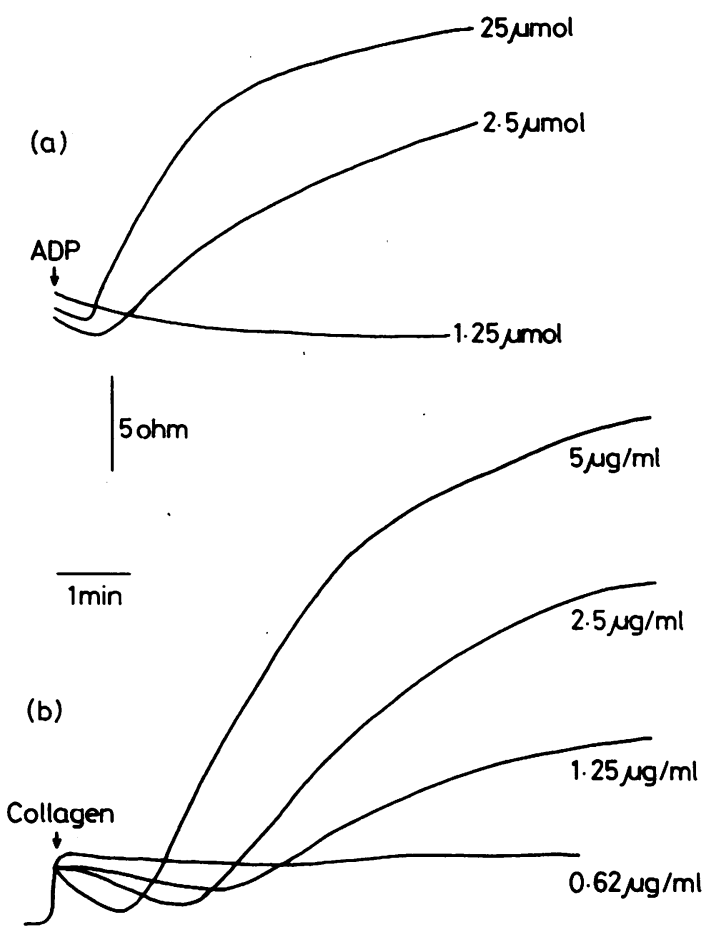

Fig. 3 Dose response curves to collagen and $A D P$. Citrated whole blood was diluted to a haematocrit of $\cdot 300$ with saline and aggregated with various concentrations of (a) ADP or (b) collagen.

same sample was tested immediately after venepuncture or up to $6 \mathrm{~h}$ after sampling. Ingestion of acetylsalicylic acid (600 $\mathrm{mg} 24 \mathrm{~h}$ before sampling) appeared to have only a small effect on collagen aggregation, while ADP responses were abolished or greatly reduced (Fig. 4).

The addition of prostacyclin immediately before aggregation led to a dose dependent inhibition of aggregation with an S-shaped dose response curve, giving an ID $_{s 0}$ of about $1 \mathrm{ng} / \mathrm{ml}$ for $5 \mu \mathrm{g} / \mathrm{ml}$ collagen.

Normal values for whole blood platelet aggregation

\begin{tabular}{|c|c|c|c|c|c|}
\hline & \multicolumn{2}{|c|}{$A D P(\mu M)$} & \multicolumn{2}{|c|}{ Collagen $(\mu g / m l)$} & \multirow{2}{*}{$\frac{\text { Arachidonate (mM) }}{1}$} \\
\hline & $12 \cdot 5$ & 25 & 1 & 5 & \\
\hline $\begin{array}{l}\text { Aggregation slope } \\
\text { Mean } \\
\text { SD } \\
\text { Range }\end{array}$ & $\begin{array}{l}45 \cdot 0 \\
18 \cdot 1 \\
13-77\end{array}$ & $\begin{array}{l}48 \cdot 6 \\
10 \cdot 9 \\
31-75\end{array}$ & $\begin{array}{l}45 \cdot 1 \\
10 \cdot 2 \\
27-62\end{array}$ & $\begin{array}{c}61 \cdot 4 \\
8 \cdot 1 \\
45-73\end{array}$ & $\begin{array}{l}46 \cdot 1 \\
11 \cdot 8 \\
27-69\end{array}$ \\
\hline $\begin{array}{l}\text { Aggregation extent } \\
\text { Mean } \\
\text { SD } \\
\text { Range }\end{array}$ & $\begin{array}{l}13 \cdot 5 \\
7 \cdot 6 \\
6 \cdot 5-40\end{array}$ & $\begin{array}{l}15 \cdot 2 \\
6 \cdot 2 \\
9-32\end{array}$ & $\begin{array}{l}13 \cdot 3 \\
4 \cdot 2 \\
6 \cdot 5-20\end{array}$ & $\begin{array}{l}22 \cdot 6 \\
4 \cdot 6 \\
13 \cdot 5-31\end{array}$ & $\begin{array}{l}20 \cdot 6 \\
12 \cdot 0 \\
2-38\end{array}$ \\
\hline
\end{tabular}

Citrated whole blood from 20 normal subjects was diluted with isotonic saline to a haematocrit of $\cdot 300$. Aggregation was performed to $12 \cdot 5$ and $25 \mu \mathrm{M}$ ADP, 1 and $5 \mu \mathrm{g} / \mathrm{ml}$ collagen, and $1 \mathrm{mM}$ arachidonate. Results are expressed as aggregation slope (degrees) and aggregation extent (ohms). 


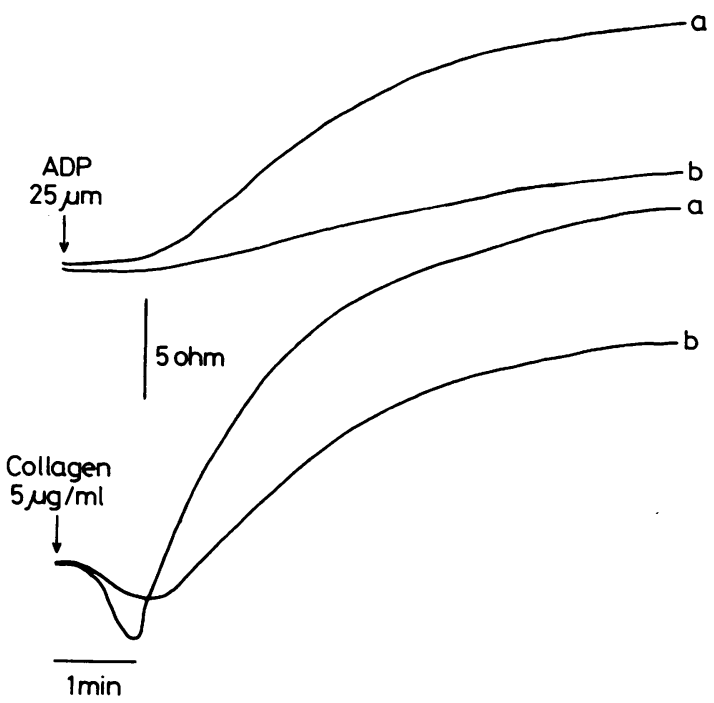

Fig. 4 Inhibition by acetylsalicyclic acid. Whole blood platelet aggregation at a haematocrit of 300 prepared with saline before (a) and $24 \mathrm{~h}$ after (b) taking $600 \mathrm{mg}$ of soluble acetylsalicyclic acid.

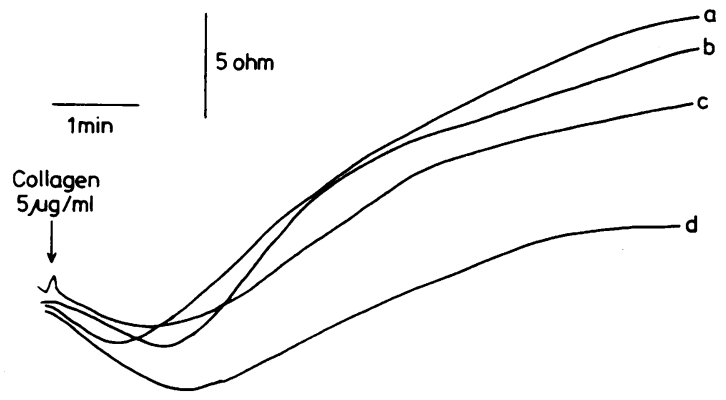

Fig. 5 Inhibition with prostacyclin. Prostacyclin (Epoprostenol) was added to citrated whole blood to give a final concentration of $1 \mathrm{ng} / \mathrm{ml}$. Aggregation was tested $(b)$ in the absence of prostacyclin and (a) $1 \mathrm{~h}, \mathrm{c}) 30 \mathrm{~min}, d) 0 \mathrm{~min}$ after adding it.

Addition of ZK 36,374 to whole blood produced a similar dose dependent inhibitory response, giving an ID so $_{\text {obout }} 1.75 \mathrm{ng} / \mathrm{ml}$ for $5 \mu \mathrm{g} / \mathrm{ml}$ collagen. Neither inhibitor was able to abolish completely the response to collagen, even with very high doses, whereas both agents could completely block ADP aggregation using relatively low inhibitor concentrations. Time course studies showed that the inhibitory effect of prostacyclin disappeared rapidly within 30-60 min after incubation with whole blood (Fig. 5 ), whereas the inhibitory effect of ZK 36,374 was stable in whole blood for at least $6 \mathrm{~h}$.

\section{Discussion}

Many of the studies of whole blood impedance aggregation have used blood from laboratory animals. ${ }^{56}$ A detailed study using human blood was therefore necessary to establish a suitable method and normal range with various aggregating reagents. One of our first observations was the dramatic influence of haematocrit on whole blood aggregation. This is of great importance owing to the wide variation in haematocrit found in clinical samples. Subjects are studied either for the diagnosis of bleeding disorders, the investigation of a thrombotic state, or the influence of antiplatelet agents. Based on our results, it seems reasonable to standardise the haematocrit to $\cdot 300$, which does not cause excessive platelet dilution and suits most cases. It is of interest that a small dilution of whole blood leads to an increase in platelet aggregation by impedance, whereas in platelet rich plasma a decrease in optical aggregation results. This increase remains fairly constant as the haematocrit is lowered, until the platelet count becomes the limiting factor. There was no correlation between response and starting haematocrit or platelet count. This effect is probably a combination of the removal of impeding red cells and the difference in conductivity between saline and platelet poor plasma.

The diluent of choice appears to be isotonic saline. This is immediately available and gives a better response than platelet poor plasma, which also requires centrifugation thus delaying analysis. Dilution with the patient's own platelet poor plasma, however, may be advisable in the investigation of certain ex-vivo inhibitory or pro-aggregatory plasma substances present in trace amounts, the effect of which may disappear due to dilution with saline.

Whole blood aggregation appeared to be more suitable than conventional aggregation for low platelet counts, giving good responses at platelet counts of about $50 \times 10^{9} / 1$; the optical technique cannot be effectively used as a diagnostic method below $100 \times 10^{9} / 1$ and in our experience is best at $200-400 \times 10^{9} / 1$. The method may therefore have important applications in the study of patients with mild thrombopenia who are bleeding.

As reported by Ingerman-Wojenski et al, ' we could not detect a reversible wave of aggregation with ADP even when a wide dose response curve was tested, and biphasic curves were not seen. Therefore responses to ADP are probably related to the nucleotide salvage pathways of red and white blood cells, which are efficient at taking up and utilising adenine nucleotides which would prevent their use by platelets. The poor response to adrenaline is difficult to explain but could also be 
due to an uptake mechanism or competition by receptors on other cells.

The weak effect of acetylsalicylic acid on collagen aggregation has been described previously ${ }^{8}$ and is believed to be due to the ability of the impedance method to detect even very small platelet aggregates which may still form in the presence of acetylsalicylic acid. Prostacyclin and the stable prostacyclin analogue ZK 36,374, however, caused a marked inhibition of both ADP and collagen aggregation, at doses of the same order of magnitude as those active in platelet rich plasma. Prostacyclin was only effective in whole blood for short incubation periods, showing the usefulness of this technique for ex-vivo studies of substances with short half lives. The discrepancy between duration of effect of prostacyclin and ZK 36,374 is probably related to the nonenzymatic degradation of prostacyclin at $\mathrm{pH} 7 \cdot 4$ to biologically inactive metabolites ${ }^{9}$; ZK 36,374 is chemically stable and although possibly adsorbed to the red cell surface does not appear to be appreciably inactivated in whole blood ex vivo. ${ }^{10}$

In summary, we recommend the following for routine clinical work: collection of blood into citrate and dilution to a haematocrit of $\cdot 300$ with isotonic saline; stirrer speed $600 \mathrm{rpm}$; $975 \mu \mathrm{l}$ blood plus 25 $\mu \mathrm{l}$ agonist (12.5 and $25 \mu \mathrm{M}$ ADP, 1 and $5 \mu \mathrm{g} / \mathrm{ml}$ collagen, 0.5 and $1 \mathrm{mM}$ sodium arachidonate); chart speed $2 \mathrm{~cm} / \mathrm{min}$; and $5 \mathrm{ohm}$ equal to $2.5 \mathrm{~cm}$ of chart paper.

The value of whole blood aggregation in the clinical laboratory remains to be seen. There may be important uses in the study of hypercoagulable states; antiplatelet treatment; and disorders of red and white cells, such as sickle cell crisis, infection, and the leukaemias. These possible applications are currently under study in our laboratory and will be reported elsewhere.
We thank Mr DF Barnard and the staff of the haematology laboratory for full blood and platelet counts; Miss J Wright for typing the manuscript; and Dr M Flood of Schering Chemicals Ltd, UK, for supplying ZK 36,374.

\section{References}

' Born GVR. Quantitative investigations into the aggregation of blood platelets. J Physiol London 1962;162:67.

${ }^{2}$ Roos H, Pfleger K. Kinetics of adenosine uptake by erythrocytes and the influence of dipyridamole. Md Pharmacol 1972; 8:417-25.

${ }^{3}$ Blackwell GJ, Flower RJ, Russell-Smith N, Salmon JA, Thorogood PB, Vane JR. Prostacyclin is produced in whole blood. Br J Pharmacol 1978;64:436.

4 Willems C, Stel HV, van Aken W, van Mourik JA. Binding and inactivation of prostacyclin ( $\mathrm{PGI}_{2}$ ) by human erythrocytes. $\mathrm{Br}$ J Haematol 1983;54:43-52.

s Cardinal DC, Flower RJ. The electronic aggregometer: a novel device for assessing platelet behaviour in blood.J Pharm Meth 1980;3:135-58.

- Bullt H, Wechsung E, Houvenaghel A, Herman AG. Prostanoids and hemostasis in chickens: Anti-aggregating activity of prostaglandins E1 and E2, but not of prostacyclin and prostaglanding D2. Prostaglandins 1981;21:1045-58.

7 Ingerman- Wojenski C, Bryan Smith J, Silver MJ. Evaluation of electrical aggregometry: comparison with optical aggregometry, secretion of ATP, and accumulation of radiolabelled platelets. J Lab Clin Med 1983;101:44-52.

${ }^{8}$ Ingerman- Wojenski CM, Bryan Smith J, Silver MJ. Difficulty in detecting inhibition of platelet aggregation by the impedance method. Thromb Res 1982;28:427-32.

' Orchard MA, Robinson C. Stability of prostacyclin in human plasma and whole blood: studies on the protective effect of albumin. Thromb Haemostas 1981;46:645-7.

${ }^{10}$ Yardumian DA, Mackie IJ, Goldstone AH, Machin SJ. The effects of a new prostacyclin analogue (ZK 36,374) on platelet function in vitro and ex-vivo. Br J Haematol 1984 (in press).

Requests for reprints to: Mr IJ Mackie, Department of Haematology, Middlesex Hospital Medical School, Riding House Street, London W1P 7LD. 